\title{
Designing an algorithm for supporting information generation process optimization
}

\author{
Tatyana E. Smolentseva ${ }^{1}$, Andrey V. Svishchev ${ }^{2}$ and Nataliya V. Katakhova ${ }^{3}$ \\ ${ }^{1}$ Federal State Budgetary Educational Institution of Higher Education "MIREA - Russian Technological \\ University", Moscow, Russia \\ ${ }^{2}$ Federal State Budgetary Educational Institution of Higher Education "MIREA - Russian Technological \\ University", Moscow, Russia \\ ${ }^{3}$ Federal State Budgetary Educational Institution of Higher Education "MIREA - Russian Technological \\ University", Moscow, Russia
}

\begin{abstract}
The paper considers the structural elements for an automated information reference decision support system (AIRDSS) in hierarchical multilevel complex organizational systems (HMLCOS). The task to ensure the functioning of the AIRDSS has been formulated. To solve it the main steps have been designated, which comprise the calculation of the importance coefficients for the supporting information (SI) elements and ordering of options based on a decision-maker's preference followed by the choice of the utility prospective one. On the basis of the steps considered, the authors propose the algorithm to form the optimal structure of the AIRDSS procedural component' elements for obtaining SI; the algorithm has a number of advantages: calculation simplicity for various experiments, a relatively simple formalization of expert knowledge into numerical values of importance.
\end{abstract}

\section{Keywords}

an automated information reference decision support system, supporting information, a decision-maker, coefficient of importance, control actions

\section{Introduction}

The main task that ensures the functioning of the automated information reference system for decision-making support (AIRDSS) is the procedural component's elements optimal structure generation to obtain supporting information (SI).

The solution to this problem is carried out in two steps:

- $\quad$ calculation of the importance coefficients $\omega$ for the SI Ion elements;

- $\quad$ solving the problem of optimizing the SI contentю

For any situation and solution, the utility function is determined [1,2] by using the theory of rational decisions, which evaluates the utility and the value of Ei in the situation $\mathrm{Sj}$ from the standpoint of the 1-th feature. Then based on the rationality postulates it is necessary to order the options according to the decision-maker's preference followed by the choice of the utility prospective one. [3]

There are no universal methods for the formation of options while analyzing the problem area of hierarchical multilevel complex organizational systems (HMLCOS), except for some models for management decision-making (MD) problems, which determine the necessary and sufficient conditions for determining all solution options.

III International Workshop on Modeling, Information Processing and Computing (MIP: Computing-2021), May 28, 2021, Krasnoyarsk, Russia

EMAIL: e-mail: smoltan@bk.ru (Tatyana Smolentseva); svishev7@mail.ru (Andrey Svishchev); Katakhova@mirea.ru (Nataliya Katakhova) (c) (i) 2021 Copyright for this paper by its authors.

Use permitted under Creative Commons License Attribution 4.0 International (CC BY 4.0).

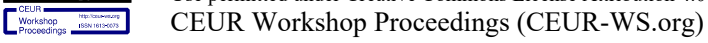




\section{Discussion}

The process of calculating the importance coefficients of the SI elements can be represented in the form of the algorithm in Figure 1:

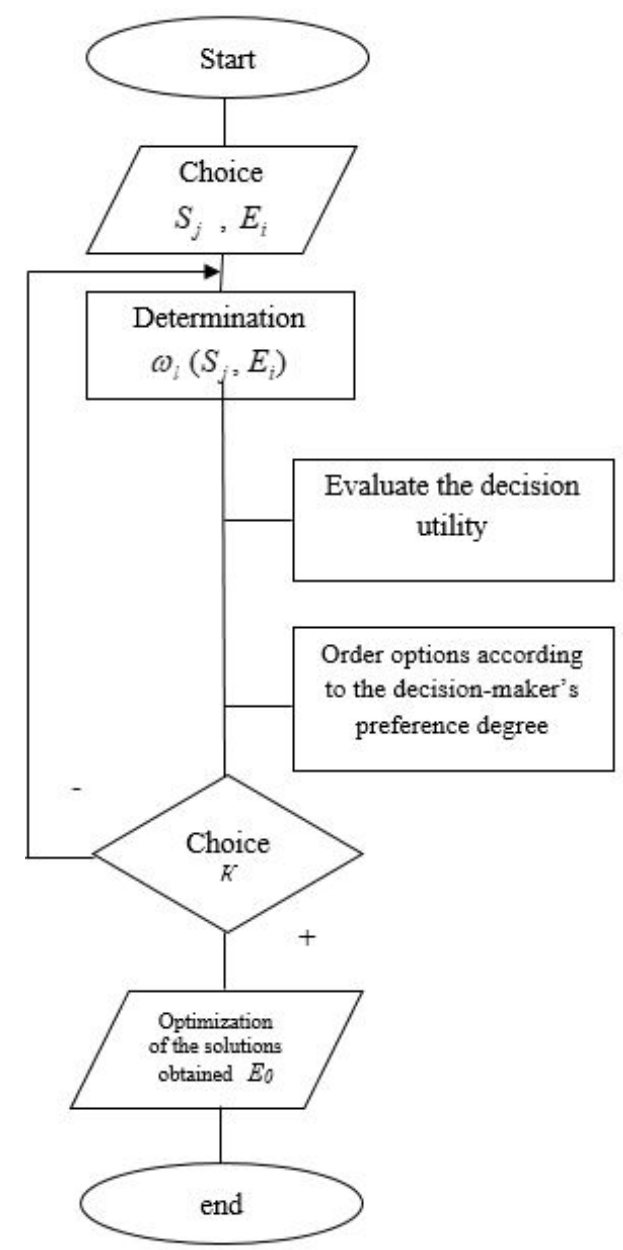

Figure 1: Algorithm for calculating coefficient of importance

For any situation $S_{j}(j=\overline{1, n})$ and decision $E_{i}(i=\overline{1, m})$ the utility function is determined $[4,5]$ using the theory of rational decisions $\omega_{l}\left(S_{j}, E_{i}\right), l=\overline{1, L}$ which evaluates the utility and the value of the decision $E_{i}$ in the situation $S_{j}$ from the standpoint of the 1-th feature. Then based on the postulates of rationality it is necessary to order the options according to the decision-maker's preference (DM) followed by the choice of the utility prospective one. [6]

Optimization of the solutions obtained is carried out on the basis of certain preferences according to the criterion $\mathrm{K}$ (or criteria) selection from the best solution point of view determined by the following expression:

$$
\max _{E_{i}} \omega\left(E_{i}\right) \Rightarrow E_{0}, \quad(i=\overline{1, m}),
$$

where $E_{o}$ is an optimal (solution) decision; $E i-i$-th decision variant; $\omega\left(E_{i}\right)$ - the value of the preference (utility) function on the i-th option.

For each i - th solution option, a number of preference functions $\omega 1$ (Ei) are formed, and the best option will be the one that meets all the criteria:

$$
\begin{aligned}
& \omega_{1}\left(E_{i}\right) \rightarrow \max , \omega_{2}\left(E_{i}\right) \rightarrow \max , \ldots, \\
& \omega_{l}\left(E_{i}\right) \rightarrow \max , \ldots, \omega_{L}\left(E_{i}\right) \rightarrow \max .
\end{aligned}
$$


Therefore, from (1) and (2), a set of possible solutions is formed, $E=\left(E_{1}, E_{2}, \ldots, E_{m}\right)$ in which there are the solutions with the remained problematic situations. The optimal solution for making management decisions (MD) is written in the form:

$$
\left\langle\mathrm{S}, \mathrm{C}, \mathrm{G}, \mathrm{E}, \omega, K, E_{0}\right\rangle \text {, }
$$

where: $\mathrm{S}$ - problematic situation, $\mathrm{C}$ - goals, $\mathrm{G}$ - constraints, $\mathrm{E}$ - solution options, $\omega$ - preferences, $\mathrm{K}$ criteria, $\mathrm{E}_{0}$ - optimal solution [7].

\section{Materials and methods}

The process of the SI formation is presented in the diagram (Figure 2:)

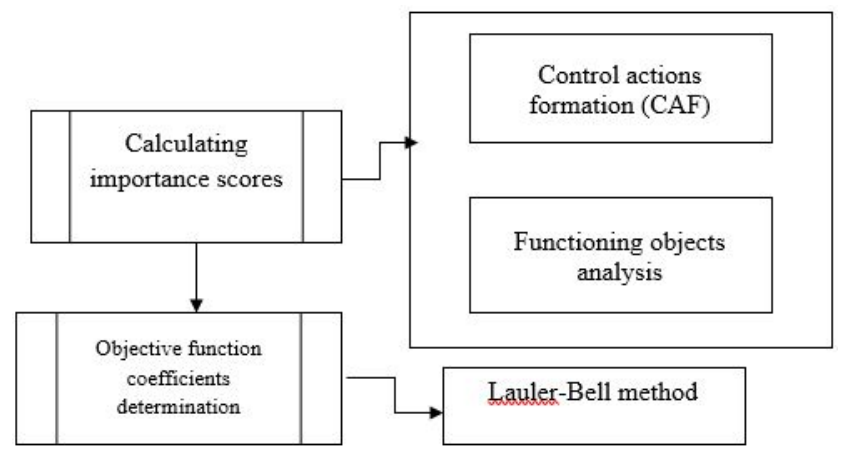

Figure 2: SI formation scheme

Step 1. To calculate the scores of importance, one can apply a number of mathematical methods, which usually relate to a specific subject area, that is why a special method must be developed for the adoption of each control action. In our case it seems desirable to apply universal methods. One of the universal methods is the method of T. Saaty $[8,9]$.

One of the main difficulties in using models for the control actions formation is the utilization of judgments identified by numerical values through a certain scale. These methods must meet many criteria, such as to reflect appropriately the subject's feelings in his judgments; the presence of judgments uncertainty should not affect the required numerical value; a large difference in judgments should reflect a significant difference on the numerical scale.

The developed model should provide close values with small deviations in the numerical representation of judgments. Comparison of two complex objects describing judgments is not so easy to carry out based on the numbers of feelings and experience about how much the influence of one of the objects affects the achievement of an assigned goal in comparison with the other one The very idea of defining numbers seems to be artificial, since it is exercised arbitrarily.

As more static data is gained, the original scale that was determined for pairwise comparisons can be adjusted and generalized. In order to form the result of the comparison of two objects in the form of objective numbers, it is necessary to conduct a more detailed analysis of these objects functioning and, in particular, how their parameters will affect the achievement of the main goal.

The source for formalizing the essence of judgments is a survey of the studied subject area experts. These judgments will determine the relative importance of one object functioning in comparison with another one in terms of the possibility of each of them to achieve its goals. Usually, when forming numerical preferences, an expert is asked the following questions: which of the two investigated objects, in his opinion, is more important; it is necessary to determine the difference on a given scale that is of greater importance. [10]

The influence of one object on another is taken into account only for those parameters that directly affect the objects functioning, and allows you to achieve the assigned goals. Consideration of the influence of indirect impacts on the objects can be carried out through the ratio of the input - output type between the objects. 
This approach is used, for example, in the distribution of energy resources between interdependent industries, taking into account their priorities. The development of the scale of the importance of objects will be carried out with the definition of the ranks of importance (Figure 3:).

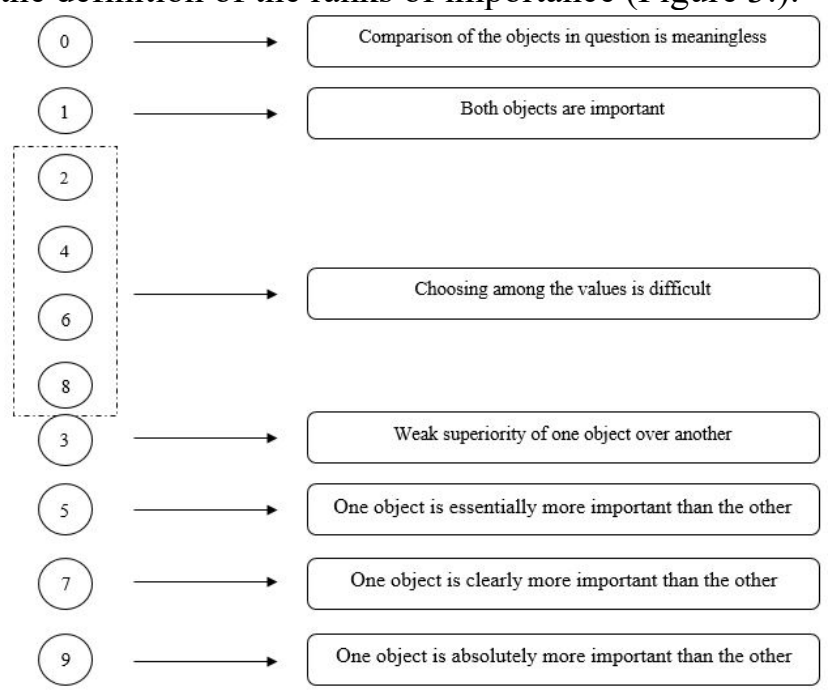

Figure 3: Relative preference scale

Let us define a set $\left(w_{1}, \ldots, w_{n}\right)$ of true values of importance for each $\mathrm{n}$-th object; on the basis of the formed scale it is possible to obtain comparative assessments of importance. Each element of the wij matrix of pairwise comparisons A forms an expert assessment of the ratio in the form of the expression $w_{i j}=w i / w j$.

While obtaining $w_{i j} w_{i k}=w_{i k}$ and, in particular, $w_{i i}=1 w_{j i}=1 / w_{i j}$, one can form a matrix. We should remember that when forming a matrix, the values obtained depend on the results of the expert judgments that are definitely difficult to formalize.

To improve the consistency of the matrix, we can recommend that the experts tried to set the value $1 / w_{i j}$ to $\mathrm{w}_{\mathrm{ji}}$, as the result of comparison the $\mathrm{i}$-th object with the $\mathrm{j}$-th one. In particular, it is preferable to define $\mathrm{w}_{\mathrm{ii}}=1$.

With this approach, assuming that one object has importance w times when compared to another, the expert will determine the importance of the second object as 1 / w of the importance of the first one. It is clear that when forming the matrix A, it has rank one, i.e.if the values of one of its lines are known, all its elements can be calculated $(w i j=w 1 j / w 1 i)$.For the matrix A, we assume that $w_{l i} \neq 0$ for all $\mathrm{i}$.

The particular case of the matrix consistency is easier to analyze, but the task will be to determine the rational ways of adopting MD regardless of the insolvency. If all judgments are determined by one or a group of experts (for example, if the knowledge of each expert is insufficient to answer all the questions). [11]

The main problem in this case is a large number of questions asked to an expert; in this case it is necessary to form $0.5 \mathrm{n}(\mathrm{n}-1)$ judgments regarding each of the defined goals when calculating reciprocal values. We assume that the required set $\left(w_{1}, \ldots, w_{n}\right)$ must satisfy the equation $\mathrm{Aw}=l_{\max }$, where $l_{\max }$ is the A's largest eigenvalue.

If the matrix $\mathrm{A}$ is non-negative and irreducible, this equation will be unique (to within a constant factor) and will have a non-negative solution $\mathrm{w}$.

If the experts formed comparative judgments and determined the first row or column, other pairwise comparisons can be used for more accurate determination of the importance of objects, since with small deviations from the consistency, stability can be increased.

If each expert from the group forms an independent comparative pair of objects, then it becomes possible to carry out multidimensional tests. Consequently, the method proposed requires to form a matrix with a list of all objects. Then, a certain goal is determined and, on the basis of all available information about the relative importance of one object in comparison with others in achieving a certain goal, the matrix elements are sequentially formed. For each goal, the process is repeated and several matrices are formed. Further, a pairwise comparison of these goals is also carried out according to their contribution to the achievement of the global goal. 
The method proposed has the following advantages:

1. It provides a relatively elementary formalization of expert knowledge (used to form A) into numerical values of importance.

2. This method is distinguished by the simplicity of calculations and, for various experiments carried out, has shown good results; for example, small changes in A lead to slight result changes.

Step 2. The problem under consideration is a linear programming problem with nonnegative coefficients in the objective function and constraints. Such problems can be solved, for example, using the Lauler-Bell method [16].

The algorithm for the AIRDSS procedural component elements' optimal structure generation to obtain SI is shown in Figure 4:

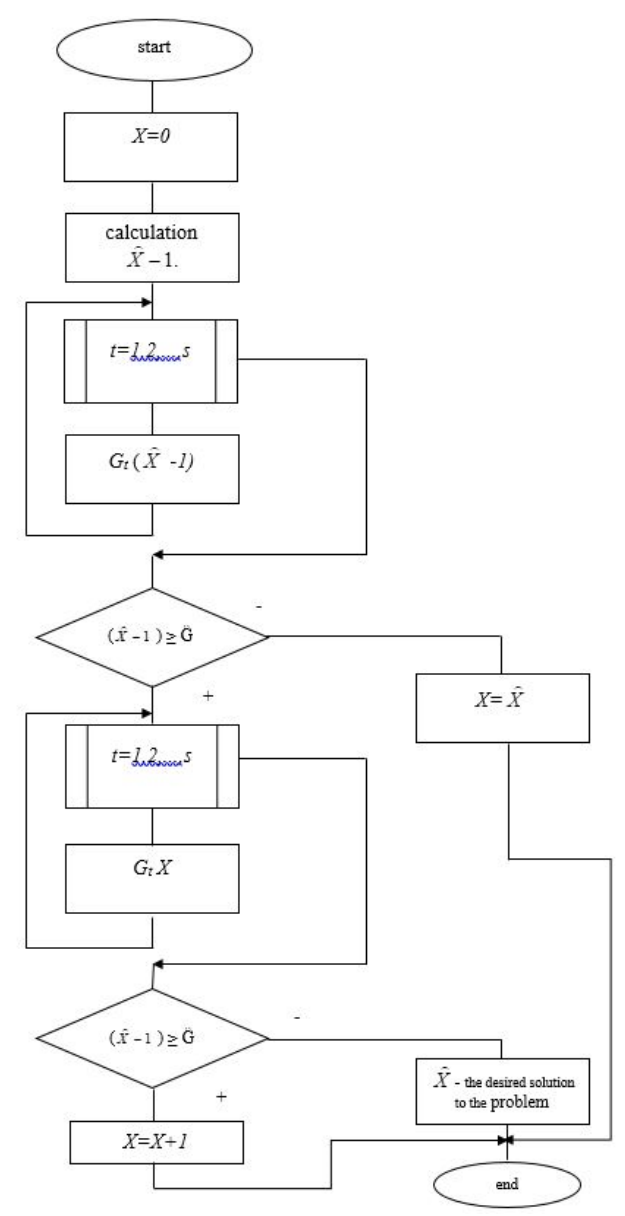

Figure 4: Algorithm for the AIRDSS structure to obtain SI

The considered algorithm for the SI generation process optimization is the basis of the process for organizational systems' (OS) multilayer hierarchy structuring, presented in the form of a set of programs: "Generation", "Approximation" and "Recursion". Building a multilayer OS hierarchy by hand is rather cumbersome. Therefore, to automate this process at the first stage, the "Generation" program has been developed.

The "Generation" program is designed to solve the following tasks:

- Determination of the graph's vertices, reflecting the structural relationship between the goals of the system function;

- Determination of the graph's arcs reflecting the relationship between the vertices of the OS graph;

- Definition of the OS goals;

- Definition of OS tasks;

- Determination of the relative volume of the work performed. 
It is possible to save a graphic image of the developed multilayer OS hierarchy in the bmp format. The structure of the program is shown in Figure 5:.

The initial data for the program when working in the mode of editing the network model of the organization's activity are:

- Expert assessments of the graph's arcs for the multilayer OS hierarchy.

- Expert assessments of the tasks of the OS multilayer hierarchy.

- Structure of the OS multilayer hierarchy.

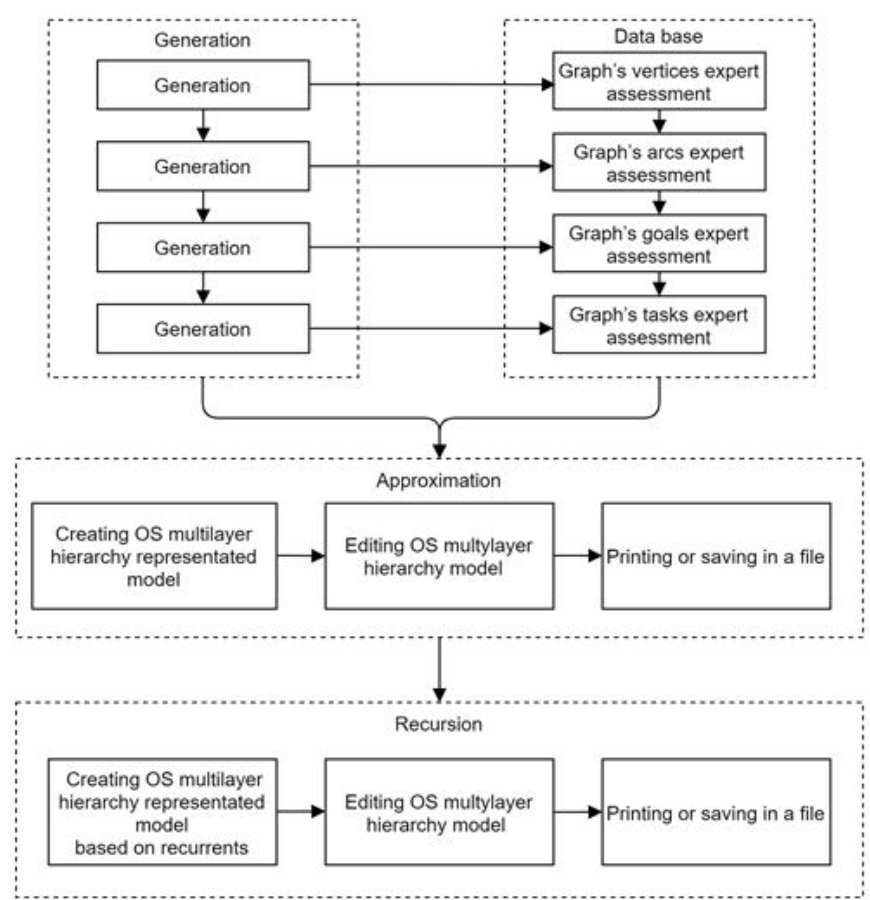

Figure 5: The structure of the set of programs for the multilayer hierarchy generation

Upon completion of the OS multilayer hierarchy generation process and processing of the expert survey data, the preliminary model for the OS multilayer hierarchy is entered into the database. The developed preliminary model for the OS multilayer hierarchy can be written into a * .bmp file. Screen forms of an example of working with the program are shown in Figure 6:, Figure 7:, Figure 8:.

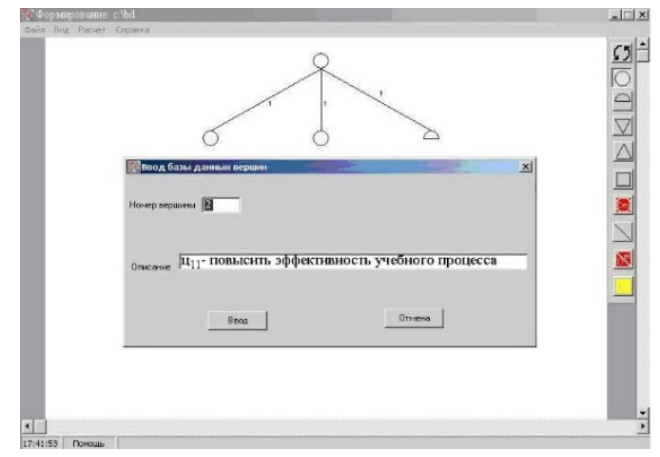

Figure 6: Entering OS multilayer hierarchy vertices 
Figure 7: Editing OS multilayer hierarchy
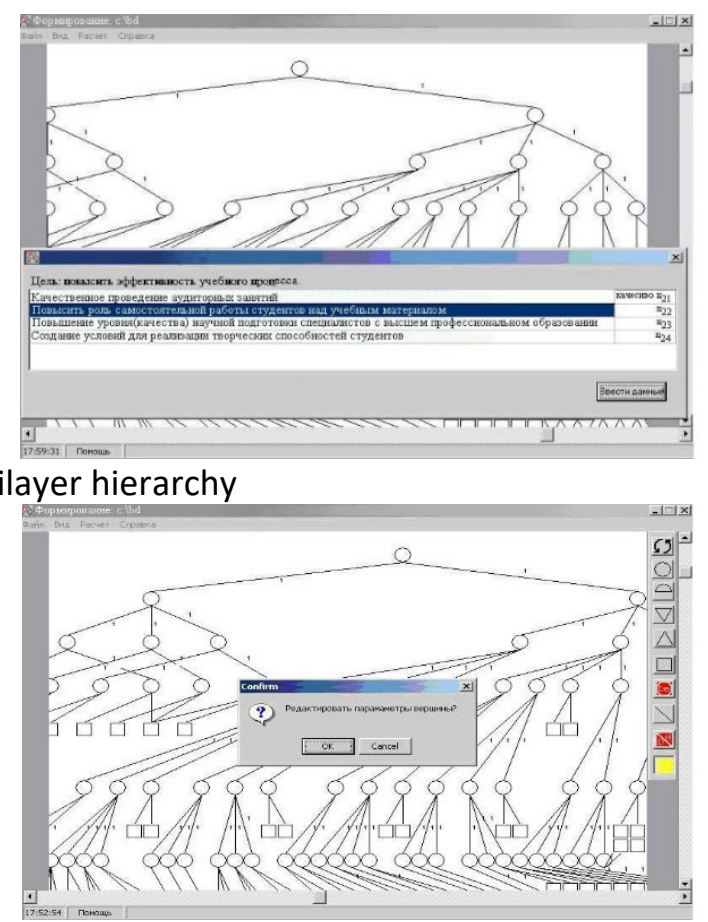

Figure 8: Editing the structure for OS multilayer hierarchy preliminary model

\section{Conclusions}

The algorithm presented is a corrected version of the algorithm for solving the linear programming problem by the Lauler-Bell method [3]. Its correctness is due to the above given property of the functionals GtX, t $=0,1, \ldots$, s. If the solution of the X problem (3) is determined, then the time for obtaining the SI is specified by the following expression:

$$
T_{0}(x)=\sum_{n=1}^{N}\left(x_{n}^{*} \sum_{i=1}^{6} a_{O n}^{i} q_{i}\left(V_{O n}^{i}, t_{O n}^{-i}\right)\right),
$$

and the total duration of the step for adopting the MD from (4) is described by (5):

$$
T(x)=\frac{V_{B}}{\bar{V}_{B}} \bar{t}_{B}+\sum_{n=1}^{N}\left(x_{n}^{*} \sum_{i=1}^{6} a_{O n}^{i} q_{i}\left(V_{O n}^{i}, t_{O n}^{-i}\right)\right)+T_{L P R} .
$$

Based on the algorithm obtained, the set of programs for a multilayer hierarchy generation is to be intended to:

- determine the goals of the organizational system,

- analyze the multilayer hierarchical structure,

- form the planning parameters system and analyze the OS functioning, - determine the standard values and coefficients of the relative importance parameters,

- $\quad$ specify the integral criteria for the evaluation of the OS functioning.

\section{References}

[1] V. I. Sumin, A. E. Nikitin, T. E. Smolentseva, Optimization of the supporting information content for the development of control actions, Modern problems of science and education 2 (2015) 194.

[2] V. I. Sumin, T. E. Smolentseva, A technique for grouping basic information for information processes in complex systems, Scientific bulletin of Belgorod State University. Series: Economics. Informatics 13(210) (2015)104-108.

[3] B. N. Ivanov, Discrete Math. Algorithms and programs, Moscow, Laboratory of fundamental knowledge, p. 288, 2000. 
[4] K. V. Baldin et al., Investments. System analysis and management, M. Dashkov and Ko, p. 288, 2018.

[5] T. E. Smolentseva, V. I. Sumin, V. P. Irkhin, R. R. Shatovkin, Justification of the management systems architecture based on hierarchical multilevel organizational systems, Bulletin of the Voronezh Institute of the Federal Penitentiary Service of Russia 3 (2017) 142-147.

[6] B. Ya. Sovetov, S. A. Yakovlev, System modeling, Moscow, Higher school, p. 343, 2015.

[7] I. P. Stabin, V. S. Moiseeva, Automated system analysis, Moscow, Mechanical engineering, p. 312, 2018.

[8] A. S. Zuev, R. G. Bolbakov, About telecommunication services based on virtual reality technologies, Russian technological journal 5(6) (2017) 3-10. https://doi.org/10.32362/2500316X-2017-5-6-3-10.

[9] V. I. Sumin, T. E. Smolentseva, Formation of the hierarchical multilevel organizational systems structure, Information technology 23(6) (2017) 476-480.

[10] B. G. Mirkin, Introduction to data analysis, Textbook and workshop, Moscow, Yurayt, p. 176, 2015.

[11] V. M. Ilyushechkin, Basics of using and designing databases, Textbook, Moscow, Yurayt, p. 214, 2015 . 